study of the surface by close fly-by and orbiting probes become available. It is not unreasonable, therefore, that an attempt should be made to summarize our present knowledge of the planet, gained mainly by Earth-based techniques. Glasstone's book provides such a summary, in a concise and detailed form, and also includes the results from the preliminary fly-by vehicle Mariner 4 .

The first chapter gives a brief outline of the more important properties of Mars and highlights many of the gaps in our knowledge of the world; the second contains an extensive history of Earth-based observation of the planet. Succeeding chapters deal, in a routine but sound way, with the astronomy of Mars, the physical properties of the surface and interior, the atmosphere, the individual types of surface feature and Martian clouds. There are particularly welcome discussions of, for example, the composition of the polar caps, the relative elevations of the dark and bright areas, and the so-called "blue haze". The "canals" are relegated to a short section on surface limeaments and cratering mechanisms; although it is true that the linear features of the Martian surface have received rather too much publicity in the past, a longer discussion of tectonic processes in the crust which could give rise to these features might well be appropriate in the present work.

Chapters eight and nine are, at first sight, something of a digression from the main theme of the book, being a fairly extensive and well organized summary of our present knowledge of molecular biology and the chemical processes leading to the formation of prebiological compounds. Their presence in this book is, however, quite justified, for the material introduced in these two chapters is used, in the next three, to explore the possibility of life on Mars, and to explain the techniques needed both to detect possible Martian life forms and to minimizo the chance of contamination of the Martian environment with terrestrial organisms.

Finally, an account is given of the methods which will be used in the next decade to explore Mars from orbiting and soft-landing vehicles.

Throughout the book, controversial issues are discussed impartially and the reader will find a fair balance of scientific opinion; a comprehensive list of references is given at the end of each chapter. The amount of mathematics has clearly been kept to a minimum, and liberal use is made of diagrams and tables - all of which are of a high standard-throughout the text. There appear to be very few errors: I noted only one, and it is of no importance. The style of the first three chapters is rather less concise than that of the rest of the book, but this is a minor detraction from the otherwise high standard of presentation. This work will certainly be of interest to the layman and should also prove useful to the scientist not specializing in planetary studies. L. WILSON

\section{ALGEBRA AND TOPOLOGY}

Linear Algebra and Analysis

By M. Zamansky. (New University Mathematies Series.) Pp. xii +466 . (Van Nostrand: Princeton, New Jersey, and London, January 1969.) 100s.

THE author of this large book has tried to encompass at least 80 per cent of the pure mathematics which the average undergraduate at university is likely to encounter. That is, starting with the elements of set theory, he proceeds through order structures, algebra, linear algebra, topology and normed vector spaces to the theory of integration. If the latter subject is the point of the book, then one must assume that the author is dissatisfied with other books in algebra and topology, and the like. The treatment of integration is generally excellent, but the preceding section on mathematical fundamentals is largely unsatisfactory for the following reasons.
As a result of oversights or misprints, there are a number of mistakes which would preclude the beginner mathematician from gaining the maximum value from the text. For instance, the example of a partition on page 14 is not a partition and the definition of a metric space on page 227 is incorrect. Also, it is easy to give a counter example to question 3 on page 21 .

The definitions are often doctrinaire rather than constructive. For instance, determinants are defined after a great wedge of exterior algebra has been ploughed through, and a topology on a set is defined by filters and fundamental families rather than by open sets. This kind of presentation leaves no room for inotivation in the mathematics.

No provision is made at the end of the book for hints and answers to the questions given at the end of each chapter. This means that any student teaching himself from the book will not be able to tell if ho is answering the examples correctly. The construction of an answers section is also good policy in the sense that it is less likely that an incorrect example such as the one mentioned here would find its way into the text.

With these criticisms in mind, one is tempted to conclude that the author could have written a much better (and cheaper) book if the initial five chapters had either been dropped or drastically pruned. 'The latter half of this book would then have formed a largely excellent treatment of the following topics in analysis: normed spaces and semi-normed spaces, the Hahn-Banach theorem, the spaces $L$ and $L^{p}$, the Lcbesgue-Nikodym and the Lebesgue-Fubini theorems and measures on the real line. R. A. FenN

\section{THEORY OF NUMBERS}

\section{Introduction to Analytic Number Theory}

By K. Chandrasekharan. (Die Grundlehren der mathematischen Wissenschaften in Einzeldarstellungen mit besonderer Berücksichtigung der Anwendungsgobiete, Band 148.) Pp. viii + 140. (Springer-Verlag: Berlin and New York, 1968.) 28 DM; $\$ 7$.

THis book consists of an account of some classical results in the theory of numbers of the kind normally included in a first course on the subject. The well known work of Hardy and Wright has long been a standard reference in this field and there are several other good texts covering more or less the same ground. But the author's clear, concise exposition and his well judged choice of argument make this new volume in the Springer yellow series particularly welcome. According to the preface we may apparently expect "a subsequent inquiry into deeper questions".

The contents of the book are fairly conventional. After establishing in the first chapter the basic results on the factorization of integers, the author turns to the study of congruences and obtains the fundamental theorems of Fermat, Euler and Lagrange. He considers next the topic of rational approximations to irrational numbers and proves, in particular, the old result of Hurwitz; the proofs involve only simple properties of Farey sequences and there is no mention of continued fractions. There follow two chapters on quadratic residues; these include expositions of the standard results on the representation of integers as sums of two and four squares and include also a discussion of Gaussian sums preliminary to a proof of the "law of quadratic reciprocity". The arithmetical functions $r(n), d(n), \mu(n), \varphi(n)$ are introduced in chapter six and their familiar multiplicative properties and elementary estimations are established. Chapter seven is devoted to a discussion of Chebyshev's results on the distribution of primes and includes, more especially, a proof of Bertrand's postulate due to S. S. Pillai. Accounts of Weyl's theorems on uniform distribution and a proof of Kronecker's theorem by the method of Bohr comprise 